\title{
Transient Science with the MWA
}

\author{
Roger J. Cappallo ${ }^{1}$ \\ MIT Haystack Observatory \\ Route 40, Westford MA, 01886 \\ E-mail: rjcehaystack.mit.edu
}

\section{Miguel F. Morales}

Massachusetts Institute of Technology

77 Massachusetts Ave., Cambridge MA 02139

E-mail: mmoralesespace.mit.edu

\section{Colin J. Lonsdale}

MIT Haystack Observatory

Route 40, Westford MA, 01886

E-mail: cjlehaystack.mit.edu

The Murchison Widefield Array is a radiotelescope, currently under construction in the outback of Western Australia, that consists of an array of 512 dipole tiles. The MWA will cover the frequency band from 80 to $300 \mathrm{MHz}$, with an instantaneous processed bandwidth of $31 \mathrm{MHz}$, and a $\sim 30^{\circ} \mathrm{FWHM}$ primary beam. Its wide field of view, excellent RFI environment, and novel software systems will allow unprecedented capabilities for discovering and observing transient radio sources. This paper describes the relevant hardware and software features of the instrument, and shows what they imply for sensitivity to various transient phenomena.

Bursts, Pulses and Flickering: Wide-field monitoring of the dynamic radio sky Kerastari, Tripolis, Greece

12-15 June, 2007

\footnotetext{
${ }^{1}$ Speaker
} 


\section{Introduction}

The Murchison Widefield Array is a low-frequency connected-element radio array, situated in the outback of Murchison Shire in Western Australia. The site was chosen principally for its pristine RFI environment (Bowman, et al., 2007), which will contribute substantially to a lower noise threshold for transient source observations. Another consideration was its moderate southern latitude $\left(\sim 27^{\circ} \mathrm{S}\right)$, where the galactic center passes nearly overhead and the entire southern sky as well as about half of the northern sky are accessible. The array is a joint project between partners in the US, Australia, and India ${ }^{1}$.

There are three key science programs to be addressed by the MWA: the Epoch of Reionization (EoR), Solar, Heliospheric, and Ionospheric science (SHI), and transient radio sources, the focus of this paper. The principal characteristics of the array, such as wide

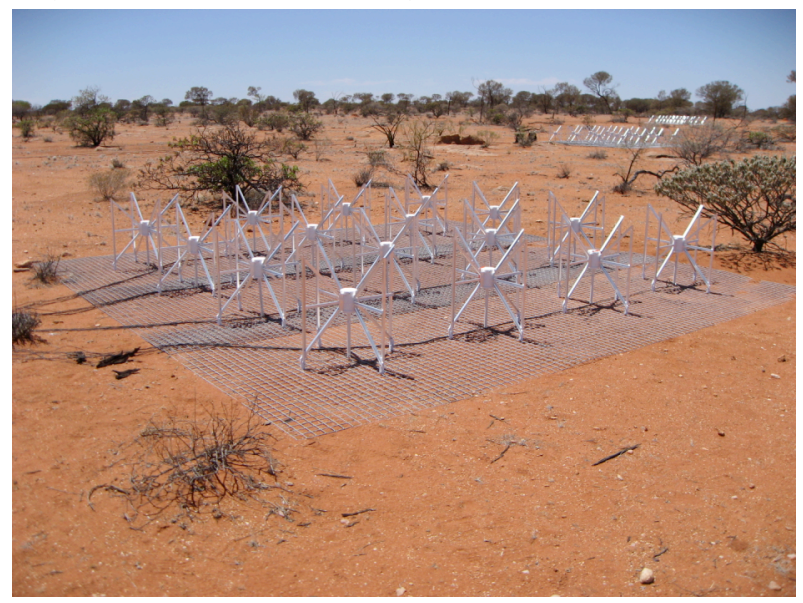
field of view, low RFI environment, and frequency range, have been chosen to serve these three key science areas, though these capabilities have been generalized as much as possible in order to cover a wide range of potential observations.

\section{MWA Instrumental Description}

The MWA consists of 8192 dual-polarization dipole antennas optimized for the 80-300 $\mathrm{MHz}$ frequency range. The antennas are arranged as a pseudo-random, centrally condensed array of 512 tiles, each of which in turn is a $4 \times 4$ array of crossed dipoles. The array has no moving parts: all telescope functions, including pointing, are performed by electronic manipulation of the dipole signals. The dipoles have a broad pattern, and contain information from $\sim 4$ steradians of sky centered on the zenith. Each tile will perform an analog beamforming operation, narrowing the field of view to a fully steerable tile beam of $\sim 1000 \mathrm{deg}^{2}$ at $150 \mathrm{MHz}$. The tiles will be spread across a circular region of $\sim 1.5 \mathrm{~km}$ diameter, in a pseudo-random centrally condensed (for high surface brightness sensitivity) pattern, forming an array with very high imaging quality, at a resolution of a few arcminutes (Figure 2).

\footnotetext{
${ }^{1}$ International consortium members include MIT and Harvard U. in the US, U. of Melbourne, CSIRO/ATNF, ANU, Curtin, other universities in Australia, and the Raman Research Institute in India.
} 
The voltage waveforms for the whole $80-300 \mathrm{MHz}$ observing band are digitized, and broken down into narrow frequency channels by two stages of polyphase filter banks. About $31 \mathrm{MHz}$ of bandwidth $(15 \%$ of the observing band) is presented to the correlator for subsequent processing. The aggregate data flow into the correlator is $320 \mathrm{~Gb} / \mathrm{s}$, carried by 192 optical fibers.
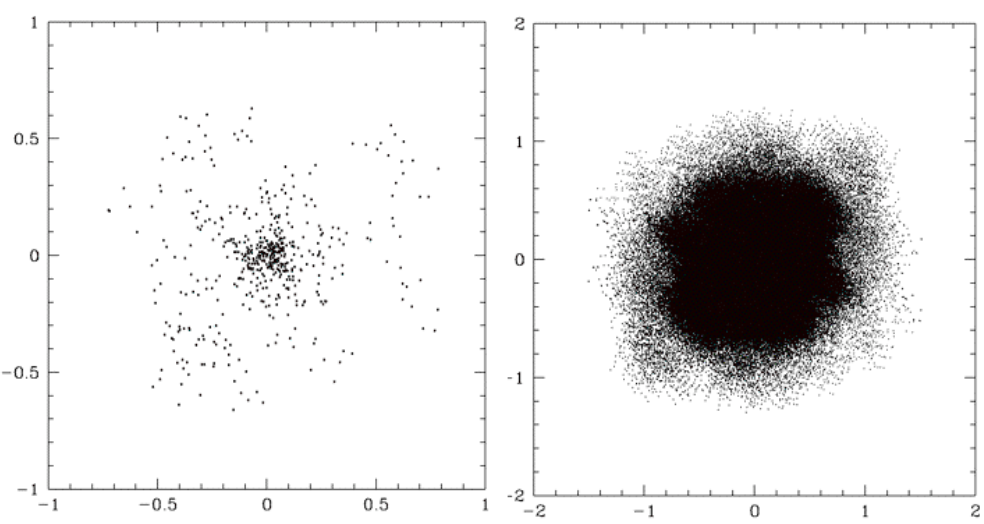

Figure 2 Typical array configuration (left) and the resulting $u v$ coverage (right). The axis units are $\mathrm{km}$.

The output of the widefield cross-correlator consists of visibilities for all $5 \times 10^{5}$ (full Stokes) signal pairs, broken into 3072 channels, and dumped every 0.5 second. The choice of a $10 \mathrm{KHz}$ channel width and a fast dump rate was made so that the intrinsic correlator field of view encompasses the full sky $(2 \pi \mathrm{sr})$, including those regions in the sidelobes of the antenna tiles. Access to the full sky allows more precise calibration, and the imaging and removal of sources outside the primary field of view.

In addition to the cross-correlator there is also a digital beamformer, which combines dual polarization voltage data from all 512 tiles, properly weighted and phased to compensate for instrumental gains, the ionosphere, and observing geometry. The beamformer output consists of 32 independently-steered, single pixel, single-polarization voltage beams, which are presented at the sample rate of the full processed bandwidth.

Software for array calibration, as well as to support specific scientific goals, runs on a small supercomputer at the site. Although the correlator is dumped every $0.5 \mathrm{~s}$, the initial computer hardware processing capability will be relatively limited, so that after fringe rotation the data will be integrated into $8 \mathrm{~s}$ segments. The visibilities will be calibrated in real time, bright sources will be removed via a "peeling" algorithm (Noordam, 2004) and the imaging software will present the resulting fully calibrated "dirty" snapshot images on an 8 second cadence. Each such image will be oversampled to about a megapixel, and appear as a data product to various science package backends. Those software packages dealing with transient data analysis will be discussed in section 4 .

\section{MWA Transient Sensitivity}

\subsection{Sensitivity}

The sensitivity of the MWA as a transient instrument depends strongly on frequency, due to the galactic background as well as the varying effective area of the dipole arrays. It also depends on the look direction: in the plane of the galaxy the thermal noise fluctuations are about 
an order of magnitude greater than near the galactic poles. The $2 \sigma$ point source sensitivity of the array, for an 8 s snapshot, centered at $200 \mathrm{MHz}$ is $7 \mathrm{mJy}$.

\subsection{Discovery Phase Space}

Due to its wide field of view, large aperture, and frequency and time resolution, the MWA will open up a significant new portion of transient discovery phase space. In Figure 3 we display that space as a function of (possibly dispersed) pulse duration and single pixel flux density. The green line denotes the $2 \sigma$ noise in a single pixel due to a pulse of specified duration, observed across the full bandwidth with an ideal filter matched to the width of the pulse. Phenomena lying above this

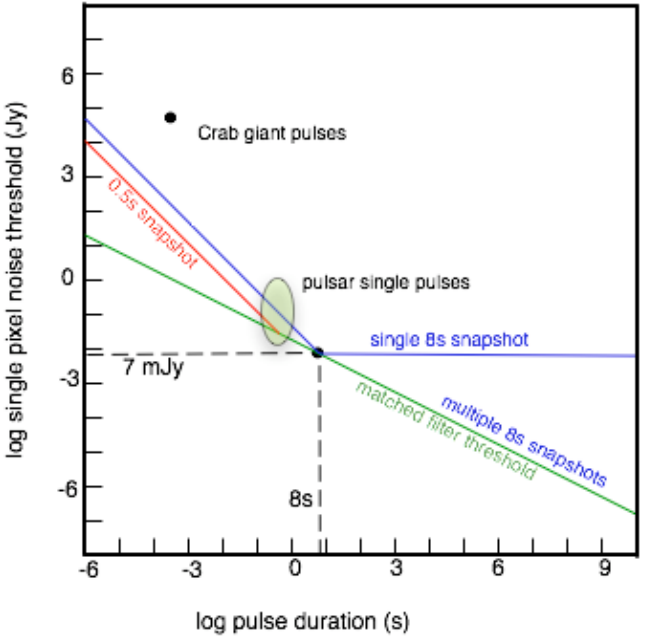

Figure $3 \mathrm{MWA}$ sensitivity as a function of pulse width (see text). line would be observable as deviations greater than $2 \sigma$. The blue line depicts the $2 \sigma$ threshold given a single $8 \mathrm{~s}$ snapshot. In the MWA we will add together multiple $8 \mathrm{~s}$ images, thus making the region down to the ideal filter accessible for $\mathrm{t}>8 \mathrm{~s}$. Also shown in red is the threshold line for $0.5 \mathrm{~s}$ snapshots, which is the hardware limit set by the correlator dump time.

\section{MWA Transient Software Analyses}

There are four backend "instruments" dedicated to transient analyses, each of which are a combination of software applications and observing programs. It is expected that each backend transient analysis tool will be used for multiple categories of transient objects. A first guess at the expected correspondence between science topics and analysis packages can be seen in Table 1. As the capabilities and limitations of the analysis tools evolve, and more is known about the prevalence and characteristics of the transient phenomena, it is expected that this mapping will change.

\subsection{All Sky Monitor}

The All Sky Monitor (ASM) consists of software (Figure 4) to perform continuous monitoring of all data flowing from the widefield correlator, in "piggyback" mode. The ASM performs extensive realtime analyses, looking for statistically

\begin{tabular}{|c|c|c|c|c|}
\hline Science Topics & ASM & TLA & Survey & BLC \\
\hline Blind Transient Search & $\mathrm{X}$ & & & \\
\hline Periodic Survey & & & $\mathrm{X}$ & \\
\hline Pulsars & & & & $\mathrm{X}$ \\
\hline Radio supernovae & $\mathrm{X}$ & & $\mathrm{X}$ & \\
\hline LIGO events & $\mathrm{X}$ & & & \\
\hline GRB prompt emission & $\mathrm{X}$ & & & \\
\hline GRB afterglows & $\mathrm{X}$ & & $\mathrm{X}$ & \\
\hline X-ray binaries & $\mathrm{X}$ & $\mathrm{X}$ & & \\
\hline Pulsar giant pulses & $\mathrm{X}$ & & & $\mathrm{X}$ \\
\hline Local gas giants & $\mathrm{X}$ & $\mathrm{X}$ & & $\mathrm{X}$ \\
\hline Extrasolar planets & $\mathrm{X}$ & $\mathrm{X}$ & & $\mathrm{X}$ \\
\hline Flare stars & & $\mathrm{X}$ & & $\mathrm{X}$ \\
\hline Scintillation events & $\mathrm{X}$ & $\mathrm{X}$ & & $\mathrm{X}$ \\
\hline Microlensing events & & & $\mathrm{X}$ & $\mathrm{X}$ \\
\hline Burpers & $\mathrm{X}$ & & & $\mathrm{X}$ \\
\hline RRAT's & & & & $\mathrm{X}$ \\
\hline
\end{tabular}

Table 1 Correspondence between transient phenomena and the planned software analysis tools 
significant changes from the previously observed sky, over a range of search parameters. The signature of the change looked for by the ASM is that of a single pulse: off-on-off. The phase space for the search will have seven dimensions:

- direction (Right Ascension and Declination)

- timing (start time and duration)

- frequency (center frequency and bandwidth)

- dispersion measure

The searches over timing, dispersion, and frequency will be performed with power of 2 binning. For example, two $8 \mathrm{~s}$ images are combined to form a $16 \mathrm{~s}$ image, two $16 \mathrm{~s}$ images are combined in a $32 \mathrm{~s}$ image, and so on, up to durations of days, with similar binning schemes for dispersion and frequency. All of these bins, as well bins staggered by half a period, are searched for significant transient signals.

The initial software will perform a blind search only over the primary field of view of the tile, but later versions will examine the sky down to the horizon, albeit at lesser sensitivity due to the tile beam gain falloff.

\subsection{Transient Lightcurve Analyzer}

The Transient Lightcurve Analyzer (TLA) allows the investigator to specify a

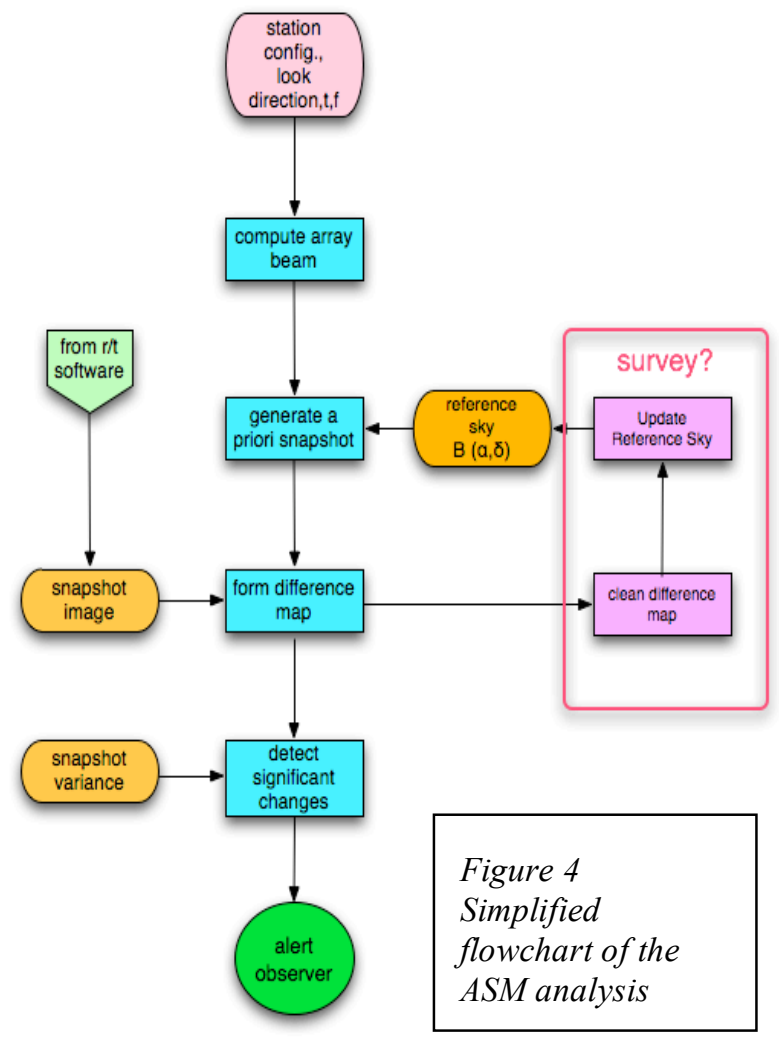
"watch list" of single pixel positions on the sky, for which light curves are desired. Whenever a desired target is within the current field of view, brightness data for the target are continually sampled and saved to disk for offline analysis. The TLA can be run either in piggyback mode, or on sources within a specified program field (such as the galactic center). In some sense, the need for the TLA arises from our inability (with current computer capabilities) to save all 8s snapshot images at full $10 \mathrm{kHz}$ resolution. By selecting only $\sim 10^{2}$ pixels in the field of view, and integrating over frequency to coarser channels, we can compress the data volume to easily manageable levels.

The principal advantage of the TLA relative to the ASM is that the offline analysis allows much more creative "filters" to be applied to the time series data. Instead of just a pulse, one can look for periodic behavior, winking, correlations with monitoring at other frequencies, etc.

A lesser advantage of the TLA is statistical in nature: Since the ASM is doing a blind search of $\sim 10^{6}$ pixels and $\sim 10^{3}$ frequency planes, it is expected that we will routinely encounter statistical outliers, whereas the TLA is examining a much smaller search space. If the pixel statistics were assumed to have a Gaussian distribution, one would expect the ASM to 
encounter about one $6 \sigma$ point per 8 second image set, and (only) two $8 \sigma$ points per year, due to the Rician distribution of the maximum. For the TLA, one would expect a $5 \sigma$ point once per year, so the effective noise floor of the TLA with a single pixel and a single frequency band, is close to a factor of two better than that of a full ASM search.

\subsection{Surveys - Short and Long Term}

The surveys are a dedicated set of periodic observations of the whole accessible sky $(\delta<$ $+35^{\circ}$ ), over the full $80-300 \mathrm{MHz}$ observing band, with offline analyses. These surveys may be conducted at two hierarchical timescales. For a short-term survey, the dwell time within each pointing and frequency band combination would be restricted to a small number of seconds and be repeated on a time scale of days. For a long-term survey, integrations of minutes can be done on monthly time scales, with more extensive image cleaning for maximum sensitivity. The surveys differ from the ASM in that only uniform sky-coverage survey data are used, and deconvolution is performed on the images.

\subsection{Beamformer Light Curve}

The beamformer light curve (BLC) analysis uses a different data product from the correlator: the output of the phased array digital beamformer. Within the BLC, high-resolution time series are formed on selected positions on the sky, saved to disk, and analyzed offline. By so doing, it is possible to get information at time scales as short as the inverse bandwidth, about $33 \mathrm{~ns}$. Since the beams need to be pointed at program sources, only known or suspected sources of transients may be observed, but the high time resolution would allow full sensitivity monitoring for very short bursts or flickers. BLC investigators will need to share the 32 available beams with the interplanetary scintillation and pulsar observing programs. Since the beams are not restricted to the primary beam of the tile arrays (albeit sensitivity will be lower in other directions), extended monitoring of sources for bright impulsive events is possible.

\section{Conclusions}

The MWA will provide a powerful new capability for the discovery and study of timevariable radio source phenomena. The dipole-based design and massive cross-correlation architecture result in a very wide effective field of view, that in principle can be used to explore a wide new discovery space. Our capacity to do this is limited by computing resources, which we will optimize via the implementation of multiple specialized software analyses, operating both on the cross-correlation output data, and on voltage-sum sample streams delivered by the digital array beamformer. The MWA will enable both blind and targeted source variability studies, over a range of timescales covering $\sim 15$ orders of magnitude from tens of nanoseconds to years. A 3.5:1 frequency range, wide fractional bandwidths and narrow channel widths will yield sensitivity to a range of dispersion measures spanning over 4 orders of magnitude. Some of these capabilities will initially be available only for targeted observations on specific pixel locations. Nevertheless, by virtue of both the array design and its radio-quiet location, the MWA will be a leading contributor to a coming revolution in our knowledge of the timevariable universe. 


\section{Acknowledgments}

This work was supported under grant AST-0121164 from the National Science Foundation.

\section{References}

[1] J. D. Bowman, et al., 2007, Field Deployment of Prototype Antenna Tiles for the Mileura Widefield Array - Low Frequency Demonstrator, AstronJ, 133:1505-1518.

[2] J. E. Noordam, 2004, Lofar Calibration Challenges, Proc. SPIE, 5489:817-825. 